\title{
Instrumentos de Avaliação da Capacidade Motora: Uma Revisão Sistemática da
}

Literatura

$\underline{\text { Júlia de Paula Silva }}{ }^{1}$; Silvia da Silva Santos Passos ${ }^{2}$; Sarah Almeida ${ }^{3}$ e Ayana Araujo de Lacerda ${ }^{4}$

1. Bolsista PROBIC/CNPq, Graduando em Enfermagem, Universidade Estadual de Feira de Santana, e-mail: silvapaulladejulia@gmail.com

2. Orientador, DSAU, Universidade Estadual de Feira de Santana, e-mail: ssspassos@yahoo.com.br

3. Bolsista do projeto de pesquisa, Graduanda em Enfermagem, Universidade Estadual de Feira de Santana, email: sarahsantosuefs@gmail.com

4. Bolsista do projeto de pesquisa, Graduanda em Enfermagem, Universidade Estadual de Feira de Santana, e-mail: ayanalacerda@hotmail.com

PALAVRAS-CHAVE: Atividades da vida diária;

Assistência à saúde; Locomoção.

\section{INTRODUÇÃO}

A integração sucessiva da motricidade implica na constante e permanente maturação orgânica. O movimento contém em si mesmo sua verdade tem uma orientação significativa em função das necessidades que o meio suscita. $\mathrm{O}$ movimento e o seu fim são uma unidade desde a motricidade fetal até a maturidade plena (NETO, 2007).

Para Teixeira (2005) a deficiência motora pode ser ainda uma alteração no corpo que provoca dificuldades de movimentação, incapacitando o indivíduo participar da vida em suas diversas dimensões, de forma independente.

O processo de incapacidade é assim representado por dimensões distintas, mas interrelacionadas. Deficiência (impairment) inclui sistemas ou partes do corpo que não funcionam apropriadamente; incapacidade (disability) centra-se em coisas que as pessoas não podem fazer, especialmente atividades básicas da vida diária e o desempenho de papéis socialmente esperados (SAMPAIO; LUZ, 2009).

A deficiência motora é caracterizada por problemas de mobilidade e coordenação motora geral em decorrência de fatores congênitos ou adquiridos como lesões nervosas, neuromusculares e osteoarticulares (BRASIL, 2008).

A maioria das deficiências motoras é derivada de condições crônicas de saúde geradoras de elevado nível de dependência de cuidados, requerendo abordagem multiprofissional (PASSOS, 2016). Desta forma, é necessário que os profissionais avaliem de forma correta a deficiência para assim contribuir com o paciente e sua família, traçando um plano de cuidado eficaz e que possa garantir maior autonomia possível.

A partir disso este estudo teve como objetivo, identificar quais os instrumentos estão sendo utilizados nacionalmente e internacionalmente para a avaliação da capacidade motora.

\section{METODOLOGIA}

Trata-se de uma revisão de literatura. A revisão da literatura é uma parte vital do processo de investigação. Aquela envolve localizar, analisar, sintetizar e interpretar a investigação prévia (revistas cientificas, livros, atas de congressos, resumos, etc.) relacionada com a sua área de estudo; é, então, uma análise bibliográfica pormenorizada, referente aos trabalhos já publicados sobre o tema. A revisão da literatura é indispensável não somente para definir bem o problema, mas também para 
obter uma ideia precisa sobre o estado atual dos conhecimentos sobre um dado tema, as suas lacunas e a contribuição da investigação para o desenvolvimento do conhecimento (BENTO, 2012).

\section{RESULTADOS E/OU DISCUSSÃO (ou Análise e discussão dos resultados)}

Durante a pesquisa foram coletadas e separadas para leitura 83 publicações relacionadas ao tema pesquisado. Dentre as mesmas foram excluídas aquelas que não atendiam ao objetivo da pesquisa, restando no fim da coleta 54 artigos para verificação do instrumento.

Os nomes dos instrumentos foram listados em um quadro onde foi possível separa-los de acordo com o ano da sua publicação, desta forma permitiu-nos melhor visualização de quais são aqueles que se repetiam com maior frequência. A partir daí identificamos a utilização de 42 formas de avaliação, entre testes e instrumentos. As formas mais utilizadas para avaliar a função motora foram o Six Minute walk test (TC6), Timed Up and Go (TUG), Motor Assentment Scale (MAS) e Medida de Independência Funcional (MIF).

Para melhor vizualição as semelhanças e divergências foram colocada em quadro comparativo (QUADRO 1). Como semelhança percebe-se que os quatro instrumentos propõem avaliar a capacidade de realizar as Atividades de Vida Diária (AVD) e tem estudos que avaliam a sua aplicabilidade e são validados para utilização no Brasil. $\mathrm{O}$ TC6, TUG e MAS são considerados instrumentos de simples aplicabilidade, pela facilidade de compreensão e de materiais para realização.

O TC6 e o TUG são realizados por meio da caminhada, já a MAS e a MIF, são instrumentos que dependem da realização de uma quantidade maior de movimentos, que devem a todo momento ser avaliados pelo profissional que está responsável por aplicar o instrumento.

O TUG, a MAS e a MIF permite que ocorra a avaliação da marcha e do equilíbrio. Além disso, a MAS e a MIF, se assemelham na avaliação da função dos membros o que não é possível nas outras formas de avaliação estudadas.

Foi possível observar que existem algumas peculiaridades na realização das avaliações realizadas com a utilização dos instrumentos estudados. O TC6 procura associar a caminhada às características hemodinâmicas e é comumente usado para avaliar pacientes com deficiência cardíaca e/ou respiratória, porém, apesar de sua alta utilização, esse teste possui como limitação o fato de não considerar a qualidade nem a velocidade de realização do movimento avaliado. Já o TUG avalia a velocidade de realização do movimento, porém a sua utilização nos estudos é direcionada principalmente para avaliação de risco de quedas em pacientes idosos.

A MAS realiza a avaliação de maior quantidade e movimentos do que o TC6 e o TUG possui a vantagem de que cada item pode ser testado de forma independente e dentre os estudos coletados é principalmente utilizada para avaliar pacientes após AVE.

A MIF é o instrumento que avalia o paciente de forma mais completa, porém isso faz com que o instrumento seja mais extenso, portanto exige maior preparo do avaliador. Além disso, outras vantagens da aplicação da MIF é que a mesma considera a cognição e determina grau de dependência, pontos esses que não são abordados em nenhum outro teste. 
QUADRO 1 - Instrumentos mais utilizados para avaliação da capacidade em pessoa

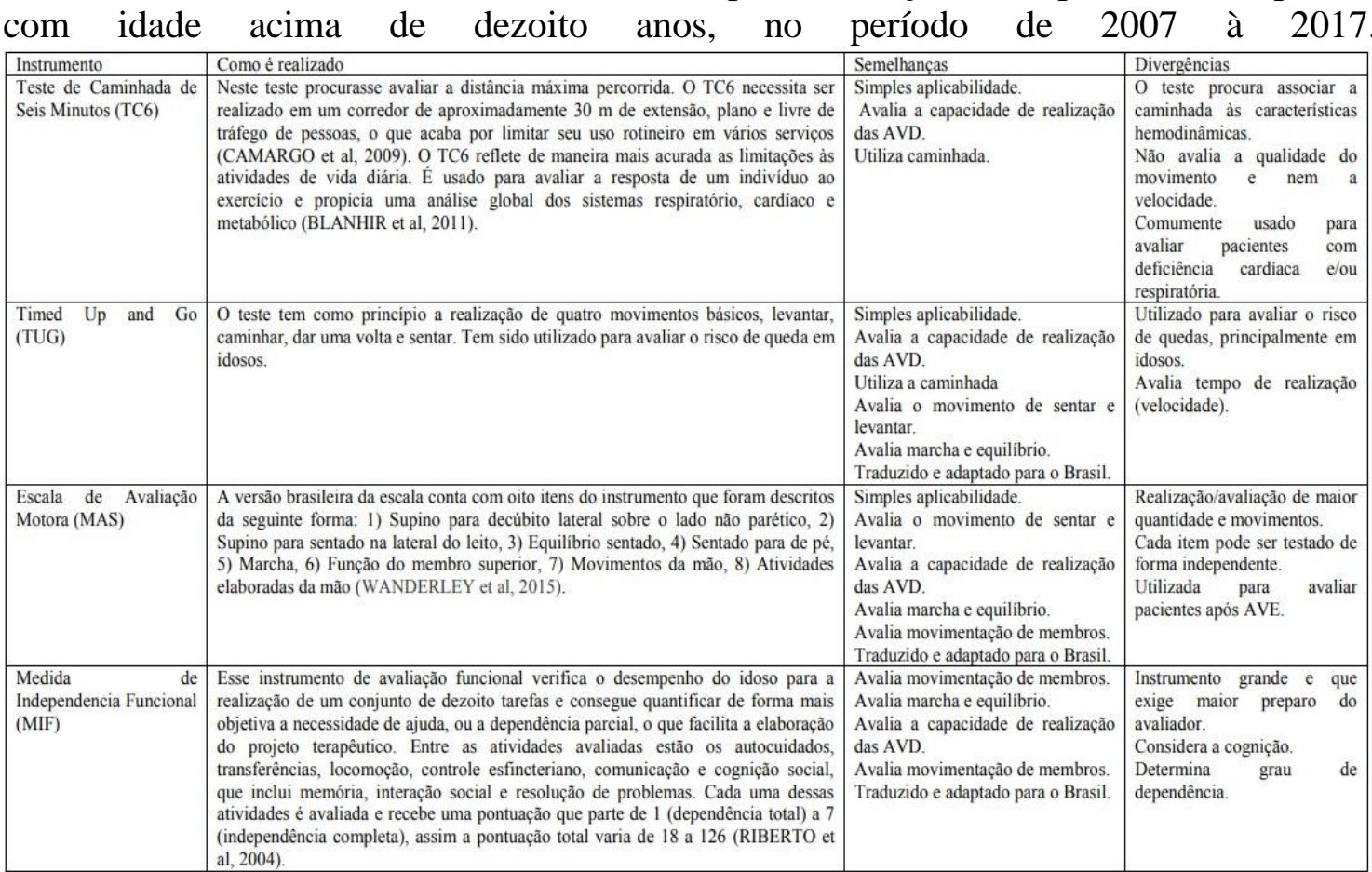

\section{CONSIDERAÇÕES FINAIS}

Com essa pesquisa foi possível notar que existem diversos instrumentos para avaliação da função motora e possibilitou conhecer quais os instrumentos que tem sido mais utilizados nos últimos anos, tendo a MIF como instrumento multidisciplinar, porém complexo. Apesar disso, tais avaliações são muitas vezes limitadas a grupos específicos, havendo a necessidade de elaboração de um instrumento que seja de aplicação mais universal e que possa ser realizados em qualquer ambiente independente da patologia apresentada.

Ao fazer a leitura dos artigos um ponto que apresentou grande relevância durante a coleta de dados foi à realização de poucos estudos no campo da enfermagem, pois a maioria das pesquisas consultadas foram feitas por profissionais fisioterapeutas e médicos.

Além disso, esse estudo apresentou como limitação a realização em apenas uma base de dados, desta forma é preciso realizar novos estudos em outras plataformas de pesquisa.

\section{REFERÊNCIAS}

BRASIL. Ministério da Saúde. Secretaria de Atenção à Saúde. Política Nacional de Saúde da Pessoa Portadora de Deficiência / Ministério da Saúde, Secretaria de Atenção à Saúde - Brasília: Editora do Ministério da Saúde, 2008.

\section{BENTO, A. Como fazer uma revisão da literatura: Considerações teóricas e}

práticas. Revista JA (Associação Académica da Universidade da Madeira), n. 65, p. 4244, mai, 2012.

NETO, F.R. Manual de Avaliação Motora. Artmed, Porto Alegre, p. 12, 2007. 
PASSOS, S.S.S. et al. Cuidado, rede de assistencia à saúde e cotidiano de pessoas com deficiência motora e suas famílias em feira de santana. Universidade Estadual de Feira de Santana. Pró-reitora de Ensino de Graduação. 2016.

SAMPAIO, R.F.; LUZ, M.T. Funcionalidade e incapacidade humana: explorando o escopo da classificação internacional da Organização Mundial de Saúde. Cad.

Saúde Publica, Rio de Janeiro, 25(3):475-483, mar, 2009. 